\title{
Fibonacci Quasiperiodic Multilayers with Internal Symmetry
}

\author{
J. P. Pandey \\ Department of Physics, M. L. K. P. G. College, Balrampur (U.P.)-271201
}

\begin{abstract}
The optical properties of quasiperiodic SFMs of $\mathrm{ZnS} / \mathrm{MgF} \mathrm{F}_{2}$ are theoretically investigated, many transmission peaks have been observed for normal incidence of light and if the symmetry is disrupted, these peaks have clearly shrunk. So, it is demonstrated that the mirror symmetry plays an important role in obtaining the perfect transmission features in SFMs.
\end{abstract}

\section{Introduction}

Recently, photonic quasicrystals (PQs) with aperiodic structures have attracted many interests for their amusing photonic band gap properties analogical to those of periodic photonic crystals. The diversity of the PBGs of PQs is magnetic both theoretically and experimentally for potential applications in novel optical and optoelectronic devices. Among various PQs, the properties of one-dimensional PQs could be simulated more precisely. So, they have attracted great attention because they are more realistic from the technical point of view. One-dimensional photonic quasicrystals including Fibonacci [1-2] and Thue Morse (TM) [3] sequences have been constructed experimentally.

The concept of a quasicrystal is a natural extension of that of a crystal, in which translational periodicity is relaxed, preserving quasiperiodic order. Quasi-periodic structures, where two (or more) incommensurate periods are superposed, can be defined as intermediate systems between a periodic crystal and the random amorphous solids [4-6]. Photonic quasiperiodic structures are deterministically generated dielectric structures with nonperiodic refractive index modulation. Quasi-periodic photonic crystals are quite different from periodic photonic crystals in many aspects [7-9]. The Fibonacci quasicrystal has been the subject of an extensive theoretical and experimental investigation in the last three decades. Merlin et al. [10] produced first Fibonacci superlattice in 1985 for electron transport studies. Kohmoto, Sutherland and Iguchi proposed an experiment using photonic Fibonacci multilayers [11] in 1987, capable of localizing photons. The transmission spectrum has a rich multifractal structure.

The photonic bandgaps of quasiperiodic photonic crystals are omnidirectional gaps. It is feasible to use quasiperiodic structures in long range of technological applications in various different fields because more structural parameters can be tuned in these as compared to periodic structures. Thue-Morse structure [12], Fibonacci sequence [13-14], Cantor layer etc. are some examples of the one dimensional quasiperiodic structures. Among them, Fibonacci sequence (FS) is a well known quasi-periodic structure and is of particular interest. The transmission spectrum of a Fibonacci system also contains forbidden frequency regions called pseudo-bandgaps similar to the band gaps of a photonic crystal. The light waves are critically localized in the frequency regime outside the Fibonacci band gaps. Hattori et al. observed the dispersion relation of photons transmitting through a photonic 1-D quasi-crystal in 1994 [15] measuring the spectrum of the phase change of the transmitted light. Gellermann et al. [16] investigated the localization of a light wave experimentally which is incident normally on a dielectric multilayer following the Fibonacci sequence. They observed a scaling of the transmission coefficient with progression through the Fibonacci sequence.

Lusk and Placido [17] designed an omnidirectional mirror from quasiperiodic dielectric stacks based on Fibonacci sequence. Dong et al. [18] reported a broad omnidirectional reflection band by combining two Fibonacci quasi-periodic structures and one periodic structure to form a heterostructures. Cheng et al. [19] calculated the density of state in a photonic quasicrystal instead of the periodic crystal, and found that there the photonic band gap existed as well.

Quasicrystals represent an intermediate organization stage between periodic dielectric materials and random media and have fascinating properties like the formation of multiple frequency band gap regions, transmission resonances and the occurrence of critically localized states. Jihene Zaghdoudi et al. have attempted to determine the optical properties of quasi-periodic symmetric one-dimensional photonic systems. In addition, it studies hybrid hetero-structure systems constructed by using periodic and quasi-periodic multilayer systems. The effect of symmetry applied to symmetric multilayer systems results in the appearance of optical windows at the photonic band gaps (PBG) of the system [20]. In this paper, the optical properties of Fibonacci $\mathrm{ZnS} / \mathrm{MgF}_{2}$ multilayers with internal symmetry are theoretically investigated. 


\section{Theoretical Formulation}

The Fibonacci sequences are multilayer structures formed by two different materials as building blocks. Two materials are labeled as $\mathrm{H}$ and $\mathrm{L}$, where $\mathrm{H}$ represents the material with high refractive index and $\mathrm{L}$ represents the material with low refractive index. The number of layers in a structure depends on the order of the Fibonacci sequence. The Fibonacci sequence can be generated by the recursive relation,

$$
\mathrm{S}_{\mathrm{j}+2}=\left\{\mathrm{S}_{\mathrm{j}}, \mathrm{S}_{\mathrm{j}+1}\right\}, \quad \mathrm{j} \geq 0
$$

where $S_{0}=H$ and $S_{1}=L$, and $S_{j}(j>1)$ is the $j^{\text {th }}$ generation of the Fibonacci structure. $H$ and $L$ are material with refractive index $n_{H}$ and $n_{L}$ and thicknesses $d_{H}$ and $d_{L}$ respectively.

The number of layers in a sequence is given by $F_{j}$, where $F_{j}$ is a Fibonacci number obtained from the recursive law $\mathrm{F}_{\mathrm{j}+1}=\mathrm{F}_{\mathrm{j}}+\mathrm{F}_{\mathrm{j}-1}$, with $\mathrm{F}_{0}=\mathrm{F}_{1}=1$. For $\mathrm{j} \geq 2$, the systems $\mathrm{S}_{\mathrm{j}}$ are known as quasiperiodic. Here, simple transfer matrix method is used to study the quasiperiodic Fibonacci structures [21, 22]. The transfer matrix for Fibonacci system $S_{\mathrm{j}}$ can be written as,

$$
\mathrm{M}_{\mathrm{j}}=\mathrm{M}_{\mathrm{j}-2} \mathrm{M}_{\mathrm{j}-1}, \quad \mathrm{j} \geq 2,
$$

with $\mathrm{M}_{0}=\mathrm{M}_{\mathrm{H}}$ and $\mathrm{M}_{1}=\mathrm{M}_{\mathrm{L}}$.

The transfer matrices for the single layer $\mathrm{H}$ and $\mathrm{L}$ are given by

$$
\begin{aligned}
\mathrm{M}_{\mathrm{H}}=\left[\begin{array}{cc}
\cos \beta_{\mathrm{H}} & -\frac{\mathrm{i}}{\mathrm{q}_{\mathrm{H}}} \sin \beta_{\mathrm{H}} \\
-\mathrm{iq}_{\mathrm{H}} \sin \beta_{\mathrm{H}} & \cos \beta_{\mathrm{H}}
\end{array}\right] \\
\text { and, } \mathrm{M}_{\mathrm{L}}=\left[\begin{array}{cc}
\cos \beta_{\mathrm{L}} & -\frac{\mathrm{i}}{\mathrm{q}_{\mathrm{L}}} \sin \beta_{\mathrm{L}} \\
-\mathrm{iq}_{\mathrm{L}} \sin \beta_{\mathrm{L}} & \cos \beta_{\mathrm{L}}
\end{array}\right],
\end{aligned}
$$

where $\beta_{\mathrm{H}}=\frac{2 \pi}{\lambda} \mathrm{n}_{\mathrm{H}} \mathrm{d}_{\mathrm{H}} \cos \theta_{\mathrm{H}}$ and $\beta_{\mathrm{L}}=\frac{2 \pi}{\lambda} \mathrm{n}_{\mathrm{L}} \mathrm{d}_{\mathrm{L}} \cos \theta_{\mathrm{L}}$ are the layer phase thicknesses. $\theta_{\mathrm{H}}$ and $\theta_{\mathrm{L}}$ are the angle of refractions in layers $\mathrm{H}$ and $\mathrm{L}$ respectively which are determined by the Snell's law and $\lambda$ is the wavelength of incident wave. Parameters $\mathrm{q}_{\mathrm{H}}$ and $\mathrm{q}_{\mathrm{L}}$ are given by, $\mathrm{q}_{\mathrm{H}}=\mathrm{n}_{\mathrm{H}} \cos \theta_{\mathrm{H}}$ and $\mathrm{q}_{\mathrm{L}}=\mathrm{n}_{\mathrm{L}} \cos \theta_{\mathrm{L}}$ for TE polarization and $\mathrm{q}_{\mathrm{H}}=\frac{\cos \theta_{\mathrm{H}}}{\mathrm{n}_{\mathrm{H}}}$ and $\mathrm{q}_{\mathrm{L}}=\frac{\cos \theta_{\mathrm{L}}}{\mathrm{n}_{\mathrm{L}}}$ for TM polarization. Thus, the transfer matrices $M_{j}$ of the Fibonacci sequences are $M_{2}=M_{H} M_{L}, M_{3}=M_{L} M_{H} M_{L}$ and $M_{4}=M_{H} M_{L} M_{L} M_{H} M_{L}$ for $S_{2}, S_{3}$ and $S_{4}$ respectively.

Here, an $N$-period finite structure is considered whose basic cell is the Fibonacci structure $\mathrm{S}_{\mathrm{j}}$. The overall transfer matrix $M$ of the system is obtained to be

$$
\mathrm{M}=\left(\mathrm{M}_{\mathrm{j}}\right)^{\mathrm{N}}=\left[\begin{array}{ll}
\mathrm{M}_{11} & \mathrm{M}_{12} \\
\mathrm{M}_{21} & \mathrm{M}_{22}
\end{array}\right]
$$

The reflection coefficient is given by

$$
\mathrm{r}=\frac{\left(\mathrm{M}_{11}+\mathrm{q}_{\mathrm{t}} \mathrm{M}_{12}\right) \mathrm{q}_{\mathrm{i}}-\left(\mathrm{M}_{21}+\mathrm{q}_{\mathrm{t}} \mathrm{M}_{22}\right)}{\left(\mathrm{M}_{11}+\mathrm{q}_{\mathrm{t}} \mathrm{M}_{12}\right) \mathrm{q}_{\mathrm{i}}+\left(\mathrm{M}_{21}+\mathrm{q}_{\mathrm{t}} \mathrm{M}_{22}\right)},
$$

where $\mathrm{q}_{\mathrm{i}, \mathrm{t}}=\mathrm{n}_{\mathrm{i}, \mathrm{t}} \cos \theta_{\mathrm{i}, \mathrm{t}}$ for TE wave and $\mathrm{q}_{\mathrm{i}, \mathrm{t}}=\frac{\cos \theta_{\mathrm{i}, \mathrm{t}}}{\mathrm{n}_{\mathrm{i}, \mathrm{t}}}$ for TM wave, where $i$ and $t$ represent incident medium and substrate respectively. The reflectivity is given by,

$$
\mathrm{R}=|\mathbf{r}|^{2} \text {. }
$$


The symmetric Fibonacci sequence can be generated in the following way. The $j$-th generation of the sequence can be expressed as- $S_{j}=\left\{G_{j}, T_{j}\right\}$, where $G_{j}$ and $T_{j}$ are Fibonacci sequences. $G_{j}$ and $T_{j}$ obey the recursion relations, $\quad \mathrm{G}_{\mathrm{j}}=\mathrm{G}_{\mathrm{j}-1} \mathrm{G}_{\mathrm{j}-2}$, and $\mathrm{T}_{\mathrm{j}}=\mathrm{T}_{\mathrm{j}-2} \mathrm{~T}_{\mathrm{j}-1}$, with $\mathrm{G}_{0}=\mathrm{T}_{0}=\mathrm{L}$ and $\mathrm{G}_{1}=\mathrm{T}_{1}=\mathrm{H}$. Therefore,

$$
\mathrm{S}_{\mathrm{j}}=\mathrm{G}_{\mathrm{j}-1} \mathrm{G}_{\mathrm{j}-2} \mathrm{~T}_{\mathrm{j}-2} \mathrm{~T}_{\mathrm{j}-1}
$$

Let us consider the symmetry in the structure as shown in the above equation and using the unitary condition $\operatorname{det}\left|\mathrm{M}_{\mathrm{j}}\right|=1$, the transmission coefficient of the light wave through the multilayers with internal symmetry can be written as,

$$
\mathrm{T}\left(\mathrm{S}_{\mathrm{j}}\right)=\frac{4}{\left|\mathrm{M}_{\mathrm{j}}\right|^{2}+4}=\frac{4}{\left[\mathrm{M}_{12}+\mathrm{M}_{21}\right]^{2}+4}
$$

If the condition $\mathrm{M}_{12}+\mathrm{M}_{21}=0$ is satisfied, perfect transmission peaks are indeed obtained. Thus, resonant transmissions can be obtained in the dielectric multulayers with mirror symmetry.

\section{Results and Discussion}

Here, the optical transmission of Fibonacci $\mathrm{ZnS} / \mathrm{MgF}_{2}$ multilayer with internal symmetry is theoretically investigated. I have chosen zinc sulphide $(\mathrm{ZnS})$ and magnesium fluoride dioxide $\left(\mathrm{MgF}_{2}\right)$ as dielectric materials A and B respectively. Around the optical telecommunication wavelength of $1550 \mathrm{~nm}$, their respective refractive indices are $\mathrm{n}_{\mathrm{A}}=2.38$ and $\mathrm{n}_{\mathrm{B}}=1.38$.
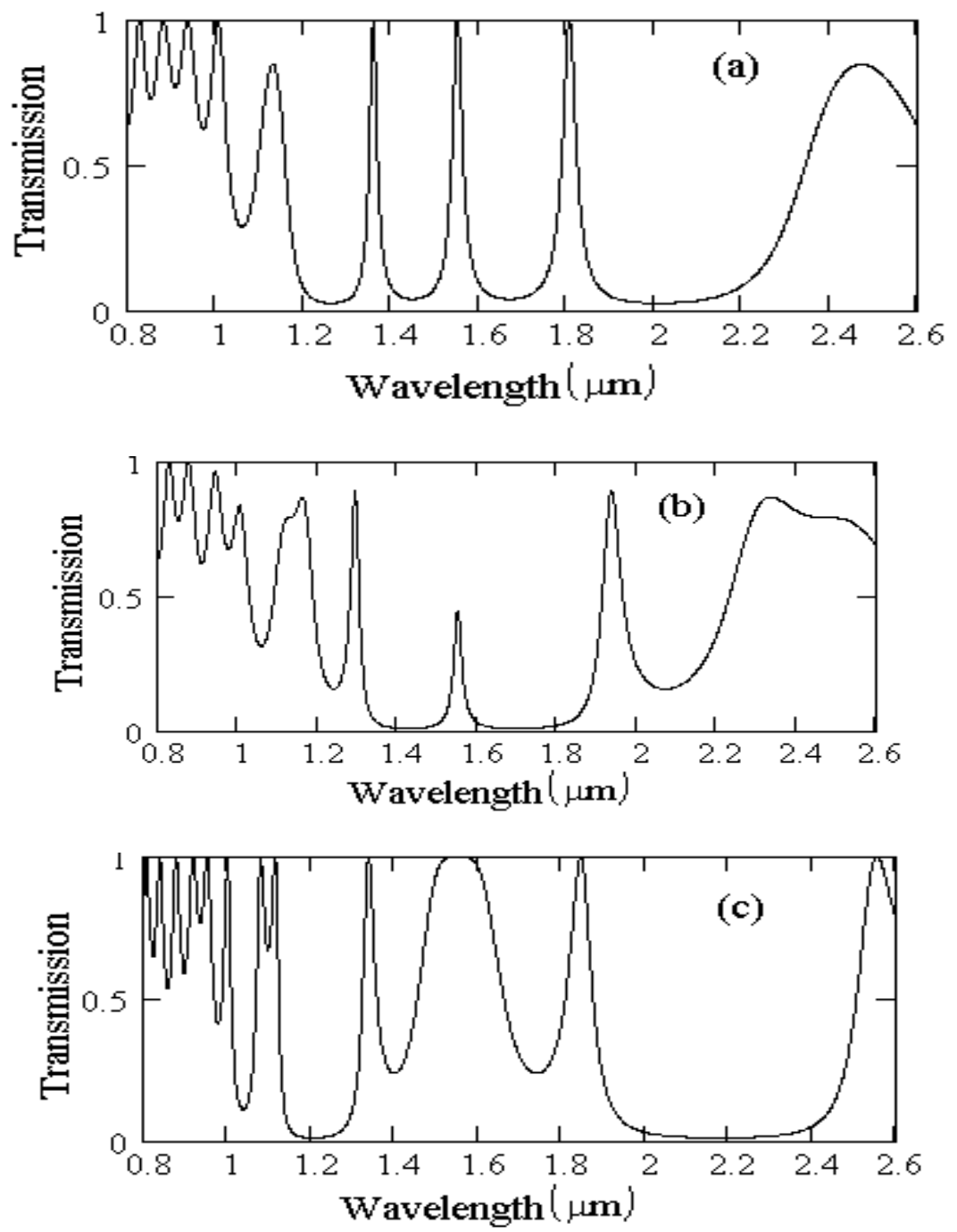

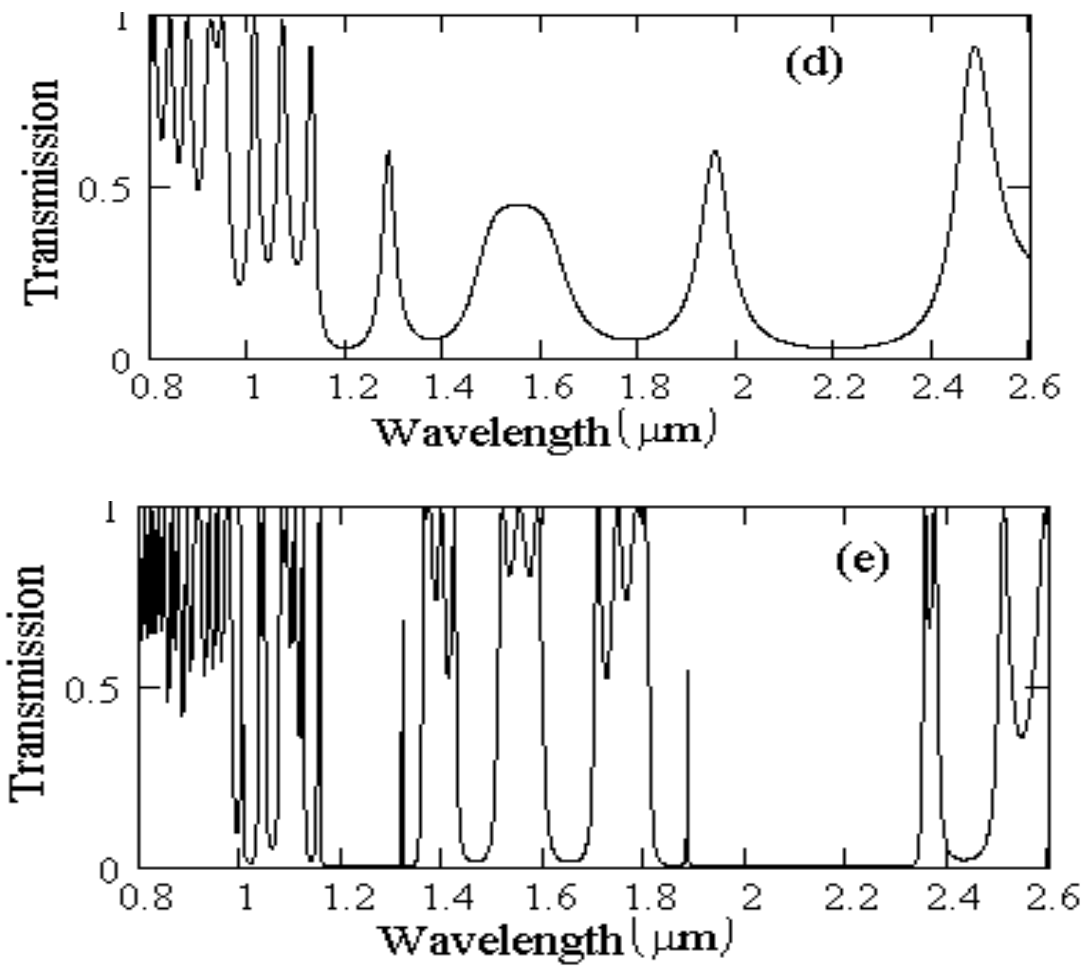

Figure 1 Calculated transmission coefficient vs wavelength for symmetrical Fibonacci $\mathrm{ZnS} / \mathrm{MgF}_{2}$ multilayers with different generations. SFM S 5 with 16 layers (a) with symmetry, (b) without symmetry; SFM S 6 with 26 layers (c) with symmetry, (d) without symmetry; SFM $\mathrm{S}_{8}$ with 68 layers (e) with symmetry.

The theoretically calculated transmission as a function of wavelength for SFM films with the generations $S_{5}, S_{6}$ and $S_{8}$ respectively are shown in the figures 1(a)-(e). Various resonant transmission peaks are observed in the wavelength range $800 \mathrm{~nm}$ to $2600 \mathrm{~nm}$. So, it can be found $100 \%$ transmission at different wavelengths by adjusting the index contrast and modulation of optical thickness. This is due to the mirror symmetry of the system which creates special structures in some transfer matrix elements making it easier to satisfy the condition of perfect transmission.

As it was seen that in the case of ordinary Fibonacci multilayer, the transmission of electromagnetic waves are poor but in the case of SFMs, perfect transmission peaks has been observed. In order to confirm it, I have deliberately disrupted the symmetry in the $S_{5}$ and $S_{6}$ systems and observed the change of the transmission spectra. In the $S_{5}$ generation, the symmetry is broken by interchanging the ninth and tenth layers. From the figures 1(a) and 1(b), it can be observed that the previous perfect transmission peaks have clearly shrunk. Also, the optical transmission changes dramatically with the loss of perfect transmission peaks if the symmetry of the generation $\mathrm{S}_{6}$ is disrupted by interchanging the $14^{\text {th }}$ and $15^{\text {th }}$ layers. These results confirm that the phenomenon of perfect transmission of light is induced by the symmetry of the internal structure in dielectric multilayer.

This work will be useful in the applications where high transmission and high resolution monofrequency outputs are particularly desired such as narrow-band optical filters, wavelength division multiplexing systems and photonic integrated circuits. These symmetric multilayer films can be used for a wide range of potential applications due to the transmittivity of resonant optical modes.

\section{References}

[1]. R. W. Peng, X. Q. Huang, F. Qiu, A. Hu, S. S. Jiang and M. Mazzer, Appl. Phys. Lett., 80, 3063-3065, 2002

[2]. R. W. Peng, Y. M. Liu, X. Q. Huang, F. Qiu, M. Wang, A. Hu, S. S. Jiang, D. Feng, L. Z. Ouyang, and J. Zou, Phys. Rev. B 69, 165109,2004

[3]. L.D. Negro, M. Stolfi, Y. Yi, J. Michel, X. Duan, L.C. Kimberling, J. LeBlanc and J. Haavisto, Appl. Phys. Lett. 84, 5186-5188, 2004.

[4]. D. Levine and P. J. Steinhardt, Phys. Rev. B 34, 596-616, 1986

[5]. P. J. Steinhardt and S. Ostlund, "The Physics of Quasicrystals", (World Scientific, Singapore, 1987).

[6]. C. Janot, “Quasicrystals: A Primer”, (Clarendon Press, Oxford, 1994).

[7]. A. Della Villa, S. Enoch, G. Tayeb, V. Pierro, V. Galdi, and F. Capolino, Phys. Rev. Lett. 94, 183903/1-4, 2005.

[8]. Y. Wang, S. Jian, S. Han, S. Feng, Z. Geng, B. Cheng and D. Zhang, J. Appl. Phys. 97, 106112/1-3, 2005.

[9]. J. D. Joannopoulos, R. D. Meade, and J. N. Winn, "Photonic Crystals: Molding the Flow of Light", (Princeton University Press, Princeton, NJ, 1995).

[10]. R. Merlin, K. Bajema, R. Clarke, F. Y. Juang, and P. K. Bhattacharya, Phys. Rev. Lett. 55, 1768 -1770, 1985.

[11]. M. Kohmoto, B. Sutherland, and K. Iguchi, Phys. Rev. Lett. 58, 2436-2438, 1987. 
[12]. L. Dal Negro, C. J. Oton, Z. Gaburro, L. Pavesi, P. Johnson, A. Lagendijk, M. Righini, L. Colocci, and D. Wiersma, Phys. Rev. Lett. 90, 55501, 2003.

[13]. F. Nori, and J. P. Rodriguez, Phys. Rev. B. 34, 2207-2211, 1986.

[14]. R. B. Capaz, B. Koiller, and S. L. A. de Queiroz, Phys. Rev. B 42, 6402-6407, 1990

[15]. T. Hattori, N. Tsurumachi, S. Kawato, and H. Nakatsuka, Phys. Rev. B. 50, 4220-4223, 1994.

[16]. W. Gellermann, M. Kohmoto, B. Sutherland, and P. C. Taylor, Phys. Rev. Lett. 72, 633-636, 1994.

[17]. D. Lusk, and F. Placido, Thin solid films 492, 226-231, 2005.

[18]. J. W. Dong, P. Han, and H. Z. Wang, Chin. Phys. Lett. 20, 1963-1965, 2003.

[19]. S. S. M. Cheng, L.-M. Li, C. T. Chan, and Z. Q. Zhang, Phys. Rev. B. 59, 4091-4099, 1999.

[20]. Jihene Zaghdoudi, Nadia Maaloul, Mounir Kanzari, Optics and Photonics Journal, 2, 270-277, 2012.

[21]. P. Yeh, “Optical Waves in Layered Media”, (New York: John Wiley \& Sons, 1988).

[22]. M. Born, and E. Wolf, "Principles of Optics”, (Cambridge: Cambridge University Press, 1998). 